\title{
Special issue on Cyberworlds 2010
}

\author{
Alexei Sourin · Daniel Thalmann • Olga Sourina
}

Published online: 1 February 2011

(C) Springer-Verlag 2011

Cyberworlds are information worlds or communities created on cyberspace by collaborating participants either intentionally or spontaneously. As information worlds, they accumulate information regardless whether or not anyone is in, and they can be with or without $2 \mathrm{D}$ or $3 \mathrm{D}$ visual graphics appearance. The topic of the cyberworlds is very essential in our time of globalization of economy and competition for resources. Cyberspace opens new waste virtual lands and unlimited opportunities for the entrepreneurs, researchers, engineers, students, and in fact to any network users. It is very common nowadays to be connected online to people and various businesses and projects around the world. We even do not think where exactly, on which server, this or that event happens or a person resides. We just say - it is in cyberspace, in Facebook, in Twitter, in Second Life, or, for computer gamers, in the World of Warcraft. Three-dimensional cyberworlds are being actively built in cyberspace and they efficiently augment, and sometimes even replace the real life. Indeed, it can be very attractive to build an office, or a shop, or even a university in a place which is free from climatic disasters, traffic problems and even property taxes. Nowadays, some countries even set up their embassies and consulate departments in shared virtual communities.

The 10th in the series, the 2010 International Conference on Cyberworlds addresses a wide range of research and de-

\author{
A. Sourin $(\varangle) \cdot$ O. Sourina \\ Nanyang Technological University, Singapore, Singapore \\ e-mail: assourin@ntu.edu.sg \\ D. Thalmann \\ EPFL, Ecublens, Switzerland \\ D. Thalmann \\ IMI-NTU, Singapore, Singapore
}

velopment topics. CW2010 accepted 49 full and 14 short papers out of 130 submitted which resulted in the following parallel paper sessions:

- Human-Computer Interaction

- Shape Modeling for Cyberworlds

- Simulation and Training

- Data Mining and Cybersecurity

- Shared Virtual Worlds and Multi-user Games

- Brain-Computer Interfaces and Cognitive Informatics

- Cyberlearning in Cyberworlds

- Computer Vision, Augmented and Mixed Reality

- Virtual Humans and Avatars

- Networked Collaboration

The papers appearing in this special issue are seven revised and extended papers which were presented at CW2010. The papers have been selected based on their reviewers' comments, quality of the oral presentations and the conference delegates' feedback. The papers address the problems of virtual characters modeling, serious games, collaboration and haptic interaction in cyberworlds.

The first paper, "Informed Character Pose and Proportion Design," presents a methodology for systematically collecting layman perception feedback, given a set of carefully chosen trait labels and character silhouette images. The authors use annotated art from shapes generated by experts and junior artists and implement a character perception game which is to gather user data for labeling characters into label clusters.

A paper "Skeleton-Aware Size Variations in Digital Mannequins" presents an efficient method to customize the size of a human body model to personalize it with industry standard parameters. With this method it is possible to generate range of different size body models by using anthropometry surveys and adapting the skeleton and motion to keep the consistency between different body layers. 
Continuing the topic of virtual characters, a new mechanism to simulate virtual human behavior is presented in a paper "An Action Selection Process to Simulate the Human Behavior in Virtual Humans with Real Personality." The proposed model can be used in applications where virtual humans should behave according to their affective state, beliefs, desires, intentions and the nature of the events they perceive from the environment.

Interest in game-based training is growing within the industry. In a paper "A Framework to Meet Didactical Requirements for Serious Game Design," an interdisciplinary work on the application of computer game principles and techniques within an automation industry training scenario is presented. An interactive simulation of a real automation plant is built as a combination of state-based and physical object behavior.

Next paper, "Fractal Dimension Based Neurofeedback in Serious Games," continues the topic of serious games. The authors propose a new nonlinear fractal dimension based approach to neurofeedback implementation targeting EEGbased serious games design. The authors illustrate their method on their EEG-based neurofeedback games that make the process of brain training more enjoyable.
A paper "An Interactive Whiteboard for Immersive Telecollaboration" presents CollaBoard: a collaboration system that gives a higher feeling of presence to the local auditory and to the persons on the remote site. By overlaying the remote life-sized video image atop the shared artifacts on the common whiteboard and by keeping the whiteboard's content editable at both sites, it creates a higher involvement of the remote partners into a collaborative teamwork.

Finally, the topic of haptic rendering and interaction in cyberworlds is discussed in "Function-based Approach to Mixed Haptic Effects Rendering." The authors propose a method for defining mixed haptic effects comprising surface, solid and force fields in virtual scenes containing various objects, such as polygon meshes, point clouds, impostors and layered textures, voxel models as well as functionbased shapes.

We are sincerely thankful to Professor Nadia MagnenatThalmann, editor-in-chief of The Visual Computer, for providing us with the opportunity to showcase the best conference papers in the area of visual cyberworlds.

Conference Co-chairs 